\title{
Invention et interprétation : chants de boisson et chants chamaniques chez les Suruí du Rondônia
}

\section{Cédric Yvinec}

\section{(2) OpenEdition}

1 Journals

Édition électronique

URL : http://journals.openedition.org/jsa/11713

DOI : 10.4000/jsa. 11713

ISSN : 1957-7842

\section{Éditeur}

Société des américanistes

\section{Édition imprimée}

Date de publication : 5 octobre 2011

Pagination : 151-177

ISSN : 0037-9174

\section{Référence électronique}

Cédric Yvinec, «Invention et interprétation : chants de boisson et chants chamaniques chez les Suruí du Rondônia », Journal de la société des américanistes [En ligne], 97-1 | 2011, mis en ligne le 10 décembre 2014, consulté le 20 avril 2019. URL : http://journals.openedition.org/jsa/11713; DOI : $10.4000 /$ jsa. 11713 


\title{
INVENTION ET INTERPRÉTATION : CHANTS DE BOISSON ET CHANTS CHAMANIQUES CHEZ LES SURUÍ DU RONDÔNIA
}

\author{
Cédric YVINEC *
}

Cet article examine les techniques rhétoriques mises en œuvre par deux genres de chants rituels des Suruí du Rondônia, chants de fête de boisson et chants chamaniques, notamment les écarts par lesquels la langue et l'énonciation rituelles se distinguent du discours ordinaire. Ces procédés rhétoriques trouvent un écho au niveau sociologique, à travers le statut du chamane et celui du grand homme dans ce groupe. [Mots-clés : Amazonie, Suruí du Rondônia, chamanisme, fête de boisson, chants rituels.]

Invention and interpretation: drinking songs and shamanic songs among the Surui of Rondonia. This article compares the rhetorical techniques of two types of ritual songs among the Suruí of Rondônia : beer festival songs and shamanic songs, with regard to their specific ways of differentiating ritual language and enunciation from ordinary discourse. These rhetorical techniques echo the sociological statuses of great men and shamans among this people. [Key words : Amazonia, Suruí of Rondônia, shamanism, beer festival, ritual songs.]

Invenção e interpretação: músicas de cauinagem e músicas xamânicas dos Suruí do Rondônia. Este artigo compara as técnicas retóricas de dois tipos de cantos rituais dos Suruí do Rondônia : cantos de cauinagem e cantos xamânicos, do ponto de vista das diferenças específicas que, em cada caso, distinguem a língua e a enunciação rituais do discurso ordinário. Estas propriedades retóricas parecem ligadas aos estatutos sociológicos respectivos do homem forte e do xamã nesse povo. [Palavras chaves : Amazônia, Suruí do Rondônia, xamanismo, cauinagem, cantos rituais.]

Dans les ethnographies amazoniennes, sont souvent distingués deux grands genres lyriques. L’un est dénommé " magique » ou « chamanique » selon des critères assez variables : tantôt parce que ces chants sont réputés efficaces, tantôt parce qu'ils recourent à une langue hermétique, tantôt aussi parce que les auteurs

* EHESS/Laboratoire d'anthropologie sociale, 52 rue du Cardinal Lemoine, 75005 Paris [cedricyvinec@yahoo.fr].

Journal de la Société des Américanistes, 2011,97-1, pp. 151-177. C Société des Américanistes. 
de ces énoncés sont des entités surnaturelles, tantôt encore parce que leur énonciation requiert l'usage d'hallucinogènes. L'autre genre lyrique reçoit la qualification, un peu par défaut, de "profane » (par exemple, Surrallés 2003, pp. 225-231) - bien que l'importance rituelle et la richesse rhétorique de ce dernier genre ne soient parfois pas moindres. Il est donc essentiel de saisir ces différences comme un système qui ne se limite pas au niveau rhétorique. Elles peuvent en effet exprimer une opposition entre différentes figures sociologiques, propre au groupe considéré, telles celles du chamane et du guerrier (Viveiros de Castro 1992, pp. 218-248 ; Oakdale 2005). C'est le cas chez les Suruí du Rondônia, un groupe d'Amazonie brésilienne parlant une langue tupi-mondé ${ }^{1}$. Ceux-ci opposent deux types d'excellence : le «savoir» (kobamne) du grand homme (ğarbaiway: " maître de la lumière »), attesté avant tout en matière politique et guerrière, et le « savoir intime » (agõa-kobamne : cœur-savoir), compétence spécifique du chamane (wãwã). Définir ainsi ce second savoir signifie, en suruí, que ses principes ne sont pas accessibles au regard d'autrui et ne peuvent être explicités. L'excellence du grand homme et la compétence chamanique sont entièrement indépendantes, elles ne s'impliquent, ni ne s'excluent mutuellement. Chacun de ces savoirs s'exprime par des chants rituels qui présentent un certain nombre de similarités formelles et de différences systématiques.

Les attitudes à l'égard de ces chants sont nettement contrastées, aujourd'hui encore, alors que leur pratique est fortement inhibée depuis la conversion au protestantisme dans les années 1990. Les chants qui attestent le «savoir» des grands hommes sont composés par ces derniers - nous les désignerons donc comme "chants humains »- et, malgré la conversion, restent volontiers entonnés par leurs auteurs ou leurs descendants. Admiratifs des chants chamaniques, œuvres immuables des esprits, et appréciant leur écoute, les Suruí n'évoquent cependant tout ce qui relève du chamanisme qu'avec la plus grande réticence et répugnent à cette pratique. L'immense majorité d'entre eux s'affirment dépourvus de ce "savoir intime »- les Suruí ne comptent actuellement parmi eux que deux chamanes.

\section{CONTEXTES D'ÉNONCIATION}

L'énonciation des chants humains comme chamaniques est toujours déterminée par des contextes rituels spécifiques. Bien que ce ne soit pas le seul, il existe un contexte idéaltypique de la production de chants humains (merewá) : les fêtes de boisson, rituel jouant un rôle sociologique important dans la régulation des tensions entre les quatre clans patrilinéaires et exogames. Lors de ces fêtes, un « maître de la bière » (ihiway), tout au long d'une année, est défié par certains de ses affins, membres d'un autre clan : il doit, avec l'aide de ses parents, produire pour ces affins de la bière de maïs $(i h)$ en quantité supérieure à ce qu'ils peuvent 
ingérer. Les producteurs de bière ne peuvent en consommer, les consommateurs (ihot) ne peuvent en produire. Les buveurs, qui affichent une hostilité provocante au début du rituel, doivent être « vaincus » par la bière que le maître les contraint à ingurgiter. Incapables de résister aux producteurs qui s'emparent de leurs armes et ornements, ils s'effondrent sous l'emprise de l'ivresse, réduits à un état de faiblesse que les Suruí comparent à un état infantile, attesté par des vomissements, des diarrhées, une démarche titubante, puis une somnolence vagissante. Seuls les buveurs masculins chantent. C'est sous l'effet de la bière que les chants humains sont produits (aparija eka: " quand on est ivre »). Le chant apparaît à la fois comme le résultat de l'ivresse débilitante et comme une proclamation de résistance, puisque le moment idoine pour chanter est « lorsqu'on est sur le point de tomber » (aar yakadeka), comme s'il s'agissait de montrer qu'on parle encore (comme un adulte) et de proclamer sa résistance face à la bière. Ces chants sont soumis à un double idéal d'originalité et de mémorabilité. Chaque buveur doit produire au moins un chant nouveau lors de chacune de ces fêtes, différent de ceux qu'il a produits précédemment et de ceux des autres buveurs. Chacun chante tour à tour son chant en dansant, les autres buveurs le reprenant en chœur. Lorsque, par la suite, un cycle rituel sera évoqué, ce sera par les chants qui y ont été produits : ils seront rechantés comme conclusion de récits, en fonction de l'aisance avec laquelle on se les remémore. De ce fait, au sein d'un groupe de buveurs-chanteurs, les chants de chacun d'entre eux seront évalués les uns par rapport aux autres : on ne retiendra que les plus mémorables, c'est-à-dire ceux qui, tout en répondant à l'idéal esthétique de cet art lyrique, se montreront les plus originaux. C'est en effet là que réside le véritable enjeu de la fête. Sous l'opposition mise en scène entre les clans exogames, par l'aspect agonistique de la consommation de bière, la fête permet en fait l'expression d'oppositions plus réelles entre des factions politiques (non nommées). Chaque faction repose sur l'association de groupes familiaux issus de deux clans distincts, qui permutent entre eux la position de producteurs et celle de buveurs d'une année sur l'autre. Ces factions rivalisent entre elles par les cycles festifs qu'elles réalisent parallèlement, de manière plus ou moins simultanée. Le prestige de ces cycles concurrents est mesuré, non seulement par le volume de bière et d'objets produits et échangés, mais aussi par la qualité des chants produits en chacun d'eux. En outre, la participation à la fête n'est pas un droit, c'est un enjeu : il faut ou bien intégrer une faction existante, qui dispose déjà d'un partenaire habituel, ou bien en constituer une nouvelle autour de soi et trouver une faction opposante-partenaire, sans quoi on sera réduit au rang peu glorieux de spectateur. La position de chanteur apparaît donc comme celle d'une soumission infantilisante à la fois recherchée - par la participation volontaire au rituel - et contestée de manière agonistique par l'acte même de chanter comme témoignage de résistance ${ }^{2}$.

Le contexte d'usage du chant chamanique est très différent. Tout d'abord parce que les chants chamaniques ne sont pas produits : chaque chant a un esprit 
chamanique (so) propre pour auteur, et le chamane ne fait que reprendre ces chants à l'identique. Cet acte énonciatif est désigné par un terme différent : non pas mere-ewá (itératif-parler) « chanter», mais mere-iga (itératif-cueillir), terme difficile à traduire, « recueillir », « comprendre », " interpréter »- mais ce n'est pas simplement « répéter » (-koe-abo-iga : voix-à.côté-cueillir) ${ }^{3}$. Les chamanes, toujours masculins, chantent soit lors de cures individuelles (palo-ma-táğa: indéfini.humain-causatif-fort), soit lors de rituels chamaniques collectifs et préventifs (so-ey-atẽ : esprit-pluriel-convoquer). Ces deux contextes s'opposent aux fêtes de boisson en ce qu'ils sont dénués de tout caractère agonistique entre groupes d'affins et de toute rivalité factionnelle : le chamane peut soigner n'importe qui, parent ou non-parent; les rituels collectifs s'adressent à la totalité du village.

Cette opposition est toutefois moins profonde qu'il n'y paraît. En effet le chamane ne se trouve jamais en position de chanteur de sa propre initiative. Il ne met jamais en œuvre ses pouvoirs à son profit, mais toujours au service d'un individu ou d'un groupe qui, de sa seule initiative, lui aura demandé d'accomplir la cure ou le rituel. Or ces demandes émanent généralement de quelqu'un qui, à l'instar du « maître de la bière » pour les chants humains, est en possession d'une ressource indispensable à l'énonciation chamanique : le tabac. Tout chamane dépend du « maître de son tabac » (ximayxoiway). Ce dernier est un homme fort à la faction duquel le chamane s'est affilié et dont il reçoit le tabac nécessaire à son activité - consommer son propre tabac serait fatal à ses pouvoirs. Le tabac produit une «ivresse » et une perte de conscience, toutes deux perçues comme identiques à celles induites par la bière - leurs effets sont dénotés par un terme unique : aparija. En outre, l'accomplissement de ces rituels, et plus encore des cures, est inévitablement une mise à l'épreuve de la compétence du chamane, comme les fêtes de bière le sont des capacités des chanteurs humains. Dans ces conditions, la position du chamane chanteur est marquée par une ambivalence homologue à celle du chanteur humain. Tout en contrôlant une technique fort utile, il se trouve placé dans une double dépendance : à l'égard des esprits auteurs des chants et à l'égard du commanditaire de leur énonciation. Le contexte d'énonciation des chants chamaniques apparaît ainsi comme une forme dérivée et complexifiée du modèle des fêtes de boisson.

\section{L'APPRENTISSAGE}

La maîtrise des chants, humains comme chamaniques, est toujours fondée sur la dénégation explicite de l'apprentissage. Il est "théoriquement » impossible d'apprendre à chanter, du moins à bien chanter. L'acquisition des chants chamaniques tient en effet de la révélation subie (soey deor oğay e : « les esprits sont venus à moi »). L'accès à cette compétence ne peut s'obtenir que par voie d'élection. Tout processus initiatique est explicitement proscrit : s'entraîner au 
chant chamanique serait « mortel », car cela susciterait la colère vengeresse des esprits. Cette révélation présente deux caractères importants. Elle est d'abord solitaire et involontaire. L'apparition des esprits ne peut survenir que lors d'un affaiblissement affectant le novice contre son gré : elle se fait souvent pendant le sommeil, à l'occasion d'un accident ou d'une maladie, et entraîne une réclusion du néophyte, qui doit rester isolé pendant plusieurs mois. S'il se met à chanter, l'élu accepte le statut de chamane par un engagement libre, mais définitif : on peut refuser de chanter, mais si l'on s'engage dans cette voie, alors on devra veiller à chanter correctement et sans relâchement, sous peine de vexation et de vindicte mortelle des esprits - ou du moins de commentaires désobligeants, accablants ou alarmants des auditeurs.

Cette révélation est en outre conçue comme abrupte, en termes de tout ou rien. Toutes les descriptions que j'ai recueillies, tant auprès d'un chamane que de non-chamanes, confirmées par celles de Mindlin (1995, pp. 115-117), insistent sur ce point : « tous les esprits » (baga so-ey : exhaustif esprit-pluriel) défilent devant le novice et lui enseignent tous leurs chants en un temps assez bref - de ce fait aucun chamane ne peut prétendre connaître plus d'esprits et de chants qu'un autre. Ce phénomène est assimilé à l'acquisition d'une «langue (-koe) étrangère » (mã-ey-e-koe: autre-pluriel-conjonction-voix). Le terme -koe, polysémique, peut ici être traduit par «langue ». En effet, il désigne une compétence générale à la compréhension et à la production d'énoncés en nombre illimité ou, du moins, très élevé. De plus, ces énoncés sont formulés dans un lexique distinct du suruí ordinaire ( pa-iter-ey-e-koe : 1 pl.incl-intensif-pluriel-conjonction-voix, « langue des humains »), violent un certain nombre de ses règles grammaticales et permettent de communiquer, d'interagir avec un ensemble déterminé d'entités, les esprits chamaniques. Enfin, ces derniers sont présumés utiliser ce -koe pour parler entre eux. Cependant, les interactions chamaniques sont toujours signalées au moyen du préfixe agõa, " cœur », qui dénote l'intériorité et l'inaccessibilité à autrui. Le seul élément de vérification de cette compétence linguistique est donc l'énonciation "correcte" des chants chamaniques. Par comparaison avec d'autres traditions chamaniques amazoniennes, la modalité d'acquisition de ces chants peut donc être qualifiée d' " anti-initiatique », en un double sens. D'abord parce qu'elle refuse qu'un individu particulier contrôle la transmission de ses compétences (par contraste, voir Déléage 2009, p. 222) - de ce fait la reconnaissance de la prétention chamanique est entre les mains du groupe ou, du moins, de l'ensemble des chamanes confirmés. Ensuite, parce que ce mode d'acquisition refuse que la compétence chamanique puisse être l'objet d'un processus d'acquisition progressif et cumulatif, admettant des degrés et des hiérarchies (par contraste, voir Oakdale 2005, pp. 78-80). Dans ces conditions, la prétention à chanter chamaniquement apparaît donc comme un coup d'éclat.

L'apprentissage des chants humains est aussi l'objet d'une dénégation, quoique de manière moins nette. Il n'existe aucune technique, aucun procédé objectivé 
et reproductible, pour produire des chants. Ceux-ci sont supposés n'être composés que dans l'instant, par une inspiration spontanée, sous l'effet de la bière. Cette capacité est considérée comme inséparable de la grandeur : il n'existe pas de processus d'apprentissage, de parcours rituel avec des étapes. Les facultés lyriques sont implicitement le résultat des actes du chanteur, un effet parmi d'autres de la majesté du grand homme ; le chant n'est pas une technique isolable et objectivable séparément des capacités à diriger, à chasser, à ouvrir des essarts et surtout à tuer, seul un tueur pouvant être un "maître de la lumière ». L'idée qu'un individu de second rang puisse, par l'effet de la réflexion, produire des chants plus beaux que ceux d'un grand homme est inconcevable pour les Suruí, car la réflexion ne peut être décrite que par des termes connotant le doute, l'irrésolution, l'impuissance, voire le déni de réalité - ces locutions signifient aussi « désirer », " imaginer », " rester figé », « rêver ».

En dépit des restrictions évoquées, il existe un certain nombre de dispositifs préparant objectivement à la maîtrise des chants - certains étant explicitement reconnus, d'autres non. Dans le cas du chant humain, on admet qu'il existe des règles et des recettes. Chacun reconnaît assez facilement qu'il « pense » à ses chants avant de les révéler dans la fête - mais on ne les répète pas avant, pour une raison simple : on risquerait de se les faire voler par des rivaux, sanction moindre, mais plus immédiate que la vengeance des esprits en cas d'entraînement au chant chamanique. Tous savent que le chant obéit à des règles. À chaque étape du cycle festif correspond un «ton » ou "genre » propre (xi-koe : 3sg-voix) - le terme -koe désigne ici, non pas la « langue » propre à un collectif stable (les humains, les esprits, etc.), mais un air et un certain nombre de thèmes et métaphores, expressément indissociables dans un contexte rituel spécifique. Ainsi, un chant de la fête dite « des paresseux » doit associer un air plutôt traînant et des allusions aux monstres peyxo, tandis qu'un chant de la fête dite « d'essartage » évoquera l'être mythologique Pic sur un air impétueux. On reconnaît enfin l'élaboration de styles individuels (yakad-ani : 3sg.imperfectif-généralité, "c'est ainsi qu'il fait »), que certains chanteurs développent progressivement et sur lesquels ils acquièrent une forme de propriété intellectuelle tacite - les imiter serait un aveu d'impuissance lyrique et de médiocrité. Ces styles s'affirment par l'exploration continue d'une veine métaphorique, qu'on filera par exemple à partir d'un mythe. Ainsi, lors de notre séjour chez les Suruí, un chanteur semblait être le seul à s'inspirer du récit du premier contact légendaire avec les Blancs dans les divers genres lyriques humains auxquels il s'essayait.

Il existe enfin une pression assez nette en faveur d'un entraînement précoce au chant humain. Les Suruí montrent une tendance prononcée à pousser les jeunes gens à s'engager dans des activités de prestige. Les hommes accomplis adoptent notamment une telle attitude dans le cadre des fêtes de bière. On désigne souvent comme leaders rituels des gens fort jeunes et peu expérimentés, parfois âgés d'à peine vingt ans et encore célibataires. Certes, ce pourrait être, de la part des gens 
au prestige déjà établi, un calcul dans les rivalités internes à la faction rituelle, qui viserait, en prenant pour partenaire un jeune, à diviser ses rivaux potentiels pour mieux régner. Il s'agit toutefois plutôt d'une tendance générale à l'« antiésotérisme ", à faire du "savoir » (kobamne) moins un secret qu'on protège qu'un spectacle qu'on offre et qu'on met au défi d'égaler - tendance homologue de l'anti-initiation du chamanisme, en ce qu'elle repose sur le refus qu'un savoir puisse être détenu et contrôlé par un individu. Ce défi de démonstration du savoir repose sur le pari que le candidat n'y réussira probablement pas parfaitement et devra reconnaître sa faiblesse. Cette tendance peut s'observer dans tout champ d'activité impliquant du « savoir », c'est-à-dire toute œuvre permettant de démontrer sa " grandeur », de la guerre à la construction de maisons : les jeunes gens sont invités à s'y lancer, quitte à devoir faire appel à des seconds expérimentés - ce qui est déjà un aveu d'incapacité. Néanmoins, c'est dans le cas des fêtes de bière et de la production de chants humains que cette propension des vieux à laisser la place aux jeunes se trouve poussée à son comble : lors d'une fête, les chanteurs les plus âgés et les plus admirés restent souvent silencieux, les jeunes hommes malhabiles étant les plus loquaces. Il y a sans doute une raison objective à cela. Alors que, dans les autres domaines, il s'agit d'objets matériellement équivalents et comparables - un meurtre en vaut un autre, une maison reste une maison -, le chant est un objet qui doit à chaque fois être différent - du fait de l'exigence d'originalité. Celui-ci peut donc être constamment rabaissé par rapport à des modèles déjà connus, soit qu'il les imite trop, soit qu'il s'en écarte trop ${ }^{4}$.

Dans le cas des chants chamaniques, tous les hommes bénéficient dès l'adolescence d'un exercice inévitable, en participant au rituel collectif où les nonchamanes reprennent en chœur certaines parties des chants chamaniques. On reconnaît par ailleurs des prédispositions claniques au chamanisme, héréditaires en ligne agnatique - il est peu surprenant que des individus fréquentant des chamanes soient familiarisés avec cette «langue ». En outre, le modèle de la révélation chamanique est bien connu de tous : chacun sait repérer certaines situations particulièrement favorables et légitimes. Aussi certaines situations typiques - morsures de serpent en particulier - suscitent-elles systématiquement des vocations chamaniques - presque tous les mordus ont connu un début de révélation. Enfin et surtout, la réclusion consécutive à la révélation fonctionne de fait comme un dispositif d'entraînement: seul, à l'abri de sa hutte, on peut chanter sans mettre à l'épreuve sa maîtrise lyrique des esprits, en déterminant librement la fréquence et l'ordre d'énonciation des chants, sans objectif assigné (à la différence d'une cure, par exemple); on peut être entendu par les autres, mais on peut feindre d'ignorer leur jugement éventuellement négatif, voire se laisser guider par leurs commentaires. Il ne semble pas rare qu'un chamane confirmé vienne discuter avec le néophyte des expériences de ce dernier, sans néanmoins rien lui enseigner. 
Du point de vue de l'apprentissage, la distinction entre chants chamaniques et chants humains apparaît donc avant tout comme une différence de degré. Dans les deux cas, apprentissage et entraînement sont impossibles ou interdits, mais il subsiste des possibilités pour contourner ces restrictions. La démonstration de son «savoir» par le chant humain comme la prétention au «savoir intime » du chant chamanique sont des ambitions qui sont libéralement proposées aux jeunes. La différence réside avant tout dans le délai et la brutalité de la sanction : le chant humain autorise des essais successifs plus ou moins heureux, alors que les chants chamaniques doivent être réussis d'emblée et ne tolèrent pas l'échec. Le chant chamanique apparaîtrait donc de ce point de vue comme une forme plus forte de chant.

\section{LE CHANT COMME FORME}

En dépit de l'opposition entre chant humain et chant chamanique, le chant comme forme possède une unité indéniable, sous ses aspects musicaux, linguistiques et stylistiques. Il s'agit toujours de chants a cappella, d'une durée d'environ 5 à 20 minutes ${ }^{5}$. Tout chant se définit aux oreilles des Suruí par une " voix » propre : un -koe, et ce, à un double niveau. Le -koe d'un chant désigne non seulement la langue où celui-ci puise son vocabulaire et sa syntaxe, mais encore une spécificité musicale et sémantique, à laquelle il doit se conformer. Tout chant humain, quoique librement composé dans la « langue ordinaire » ( pa-iter-ey-ekoe : 1pl.incl-intensif-pluriel-conjonction-voix), doit relever d'un " genre » ou «ton » (xi-koe : 3sg-voix) associant un air précis, un noyau de thèmes et métaphores (i.e. un ensemble d'instruments signifiants) et un contexte rituel particulier (donc un champ d'énoncés pertinents relativement restreint). Pour les chants chamaniques, la notion de -koe désigne, outre la « langue » commune à tous les esprits (so-ey-e-koe: esprit-pluriel-conjonction-voix), l'«air» musical ou le « ton » propre à un chant et à son esprit auteur (ce qui restreint le champ des significations qu'on peut inférer de ce chant aux caractères spécifiques à son auteur - par exemple son appartenance au monde aquatique, terrestre ou céleste, son mode d'action, ses intentions bienveillantes ou non, etc.).

Linguistiquement, tout chant, humain ou chamanique, exige des modifications par rapport à la langue ordinaire. La plus importante consiste dans l'usage systématique de la particule évidentielle ya. Celle-ci a ordinairement une valeur non-testimoniale (ouï-dire ou non-assertif, contrastant avec de, testimonial et emphatique). Bien qu'aucune motivation ne puisse en être apportée par les informateurs, il s'agit là d'un principe que certains informateurs peuvent formuler explicitement : tout chant qui ne la respecterait pas serait jugé incorrect. Cet usage est pourtant paradoxal : il s'agit de chanter sur des faits déjà réalisés - l'ingestion de bière - dont on est par excellence l'auteur et donc, pourrait-on 
présumer, le témoin. Dans le cas du chant chamanique, le mode non-testimonial pourrait certes dénoter la distinction entre l'énonciateur humain et l'esprit auteur du chant ; mais, là encore, le chant est considéré comme le discours direct de l'esprit, qui y décrit ses faits et gestes ${ }^{6}$. Bien que sa valeur signifiante soit restreinte par son caractère obligatoire, cet usage ne peut manquer de créer un effet d'implication des auditeurs, puisqu'ils se trouvent potentiellement inclus parmi les auteurs de ces énoncés, placés sous l'autorité d'un « on dit » général et indéterminé. Cela induit un dédoublement ou une dispersion de l'énonciateur du chant, dégageant partiellement le chanteur de ses affirmations (Desclés et Guentchéva 2000) tout en y impliquant virtuellement le destinataire, ce qui interdit toute détermination simple et univoque de la vérité et de la légitimité de ces énoncés.

C'est d'un point de vue stylistique que la similitude structurale la plus importante se manifeste : tout chant est construit sur une structure paralléliste, opposant un leitmotiv à des développements. La distinction constante/variable y dépend toutefois du point de vue qu'on adopte. Au sein d'un chant, le leitmotiv constitue par définition un élément constant, tandis que les développements procèdent par ajout de variations. Entre plusieurs chants, en revanche, les leitmotive constituent les variables permettant de distinguer un chant d'un autre, tandis que les développements présentent de nombreuses récurrences d'un chant à l'autre, au sein d'un même genre.

\begin{tabular}{l|l|l} 
& Leitmotiv & Développements \\
\hline Au sein d'un chant & Constante & Variable \\
\hline Entre plusieurs chants & Variable & Constante
\end{tabular}

On peut constater cette relative invariance des développements en comparant deux à deux ces extraits de chants humains (A et B), produits par deux frères pour fêter la construction d'une maison, où les leitmotive sont en gras et les constantes des développements sont soulignées :

(A) homme de 40 ans, chant composé et recueilli en 2005

Owener axiter, owener axiter a, ikõrey xi $i$

Owener axiter, ikõrey xa mammóyheperẽkabi ter asabakãhresahraã kaled $i$, tasa ye $i$

Owener axiter, owener axiter a, ğamebey xi $i$

Owener axiter, ğamebey $x a$ mammóyhekãyataga ye $i$, maloypóyhpami beka ye $i$, tasa ye $i$

Ãter bo pala maloypóyhpami beka palade pamawatãrapataga te ani ena ana po yedekoy aye, la kaled $i$
C'est moi qui le dis vraiment, c'est moi qui le dis vraiment, disent les aigles-harpies, n'est-ce pas

C'est moi qui le dis vraiment, disent bien les aiglesharpies en apportant l'ossature de leur maison jusqu'à la fourche du grand châtaigner du Brésil, disent-ils, n'est-ce pas

C'est moi qui le dis vraiment, c'est moi qui le dis vraiment, disent les guêpes noires, n'est-ce pas

C'est moi qui le dis vraiment, disent les guêpes noires en perçant le grand châtaigner sec, par peur du déluge, n'est-ce pas

C'est ceci que nous faisons par peur du déluge, nous perçons notre arbre sec, disent-elles bien, n'est-ce pas 
(B) homme de 45 ans, chant composé et recueilli en 2005

Kanater ğamebey ăga kaytxer

Kanater ğamebey ăga kaytxer amawatãrapaã, te ikayã boliyã, te oliyã

Kanater ikõrey ăga kaytxer

Kanater ikõrey ăga kaytxer mammóyheperẽkabi ter amaipeneposahraã asabakãramoga ikayã, te boliyã
Sont-elles nombreuses les guêpes noires

Sont-elles nombreuses les guêpes noires à arranger leur arbre sec, lui demandé-je, demandé-je, dit-on

Sont-ils nombreux les aigles-harpies

Sont-ils nombreux les aigles-harpies à apporter leurs branches jusqu'à la fourche du grand châtaigner du Brésil pour rassembler l'ossature de leur maison, lui demandé-je, dit-on

Cette stabilité des développements s'observe à l'identique entre ces deux extraits de chants chamaniques d'esprits aquatiques ( $C$ et $D)$, distingués par leurs leitmotive :

\section{(C) Esprit Ğorpatih, " Crabe », chant recueilli en 2006}

Soleba araaki, soleba araaki wa

Soleba araaki, soleba araaki a ya

Enama yepere yeka te te oya tiga pamaihkinima ayugey yexipob yemãtẽ labaeykanarpõ

Soleba araaki, soleba araaki a ya

Soleba araaki, soleba araaki wa

Enama yepere yeka te te oya tiga pamaihkinimaamadĩ pamaiporkãypabepesahr ã
Les touffus arrivent, les touffus arrivent, oui

Les touffus sont arrivés, les touffus sont arrivés, dit-on

Tu m'as invité à faire cela et à ce moment-là même, marchant sur notre eau immense et infinie, je suis arrivé à ton invitation pour les gens des maisons en chantant ce que je chante maintenant

Les touffus sont arrivés, les touffus sont arrivés, dit-on

Les touffus arrivent, les touffus arrivent, oui

Tu m'as invité à faire cela et à ce moment-là même en face de notre eau immense j'étais une plaque d'écume large d'une brasse

(D) Esprit Wabewewãrtih, « Celui qui reste dans la rive », chant recueilli en 2006

\section{Goyaney jari, ğoyaney jari $i$}

Goyaney jari a ya

Enama yepere yeka te te oya tiga pamaihkinima ayugey yexipob yemãtẽ tiga yoikãr ğasopob yemãtẽ labaeydawera

\section{Ğoyaney jari, ğoyaney jari $i$}

\section{Goyaney jari a ya}

Enama yepere yeka te te oya tiga pamaihkinima ayugey yexipob yemãtẽ tiga pamaihkinimaamadĩ pamaiporkãypabepesahr ã

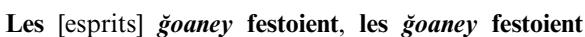
bruyamment, n'est-ce pas

Les ğoaney festoient bruyamment, dit-on

Tu m'as invité à faire cela et à ce moment-là même, alors que les palmes d'eau marchaient bruyamment, marchant sur notre eau immense et infinie, je suis arrivé à ton invitation au milieu des gens des maisons

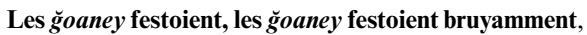
n'est-ce pas

Les ğoaney festoient bruyamment, dit-on

Tu m'as invité à faire cela et à ce moment-là même je suis arrivé marchant sur notre eau immense et infinie et alors même, en face de notre eau immense, j'étais une plaque d'écume large d'une brasse

Leitmotive et développements permettent chacun de démontrer des compétences complémentaires. Les développements attestent toujours d'une compétence générale, tandis que les leitmotive relèvent d'une mise en œuvre particulière. Dans un chant humain, les développements certifient la capacité à produire un chant conforme aux lois du genre, car il faut respecter l'air et les 
métaphores le définissant; le leitmotiv est le lieu de l'inventivité, où doit se dévoiler l'excellence singulière du chanteur. De même, dans les chants chamaniques, la capacité à conduire des développements démontre la maîtrise de la «langue des esprits » (soeyekoe), tandis que la mobilisation de nombreux leitmotive apparaît comme l'indice de la puissance du chamane - ou, du moins, de sa bonne volonté conjoncturelle, dans telle cure ou tel rituel - à convoquer de nombreux esprits.

Il existe enfin une certaine unicité des principes d'évaluation esthétique. La valeur esthétique du chant se concentre toujours dans le leitmotiv. Dans les chants humains, c'est par définition le leitmotiv qui permet le plus de liberté et d'inventivité mélodique et sémantique. Plus encore dans les chants chamaniques, où les développements suivent tous le même air, le leitmotiv constitue l'identité du chant et de l'esprit. Enfin les pratiques de chœur portent presque exclusivement sur les leitmotive - seuls ceux-ci sont repris en chœur par les gens ordinaires dans les rituels chamaniques. Il existe en outre une échelle de valeur esthétique commune aux chants humains et chamaniques. Ces derniers sont en effet explicitement désignés comme le degré suprême de l'activité lyrique et l'étalon de sa « beauté » (paor). Cela n'a rien de fortuit si l'on considère que le chant humain aspire à la longévité mnémonique : les chants chamaniques ne sont-ils pas répétés à l'identique depuis toujours?

\section{LA LANGUE DES CHANTS CHAMANIQUES}

En dépit de la reconnaissance esthétique dont ils jouissent, les chants chamaniques sont considérés par les gens ordinaires comme des discours relativement incompréhensibles (kobahr : « ignorer »), particulièrement dans leurs leitmotive. Ils restent cependant des énoncés dotés d'une signification, potentiellement traduisibles en langue ordinaire. Une telle « traduction » systématique des chants chamaniques en suruí ordinaire permet de mettre en évidence un certain nombre de procédés d'obscurcissement du sens, qui peuvent être classés en trois types : linguistiques, logiques, sémantiques.

Les premiers de ces procédés affectent la compréhension à un niveau purement linguistique, de manière plus ou moins radicale. Au niveau phonologique, on note des déformations consonantiques : le terme ğannih, « ciel », devient ainsi kattih. On repère aussi des phénomènes de métanalyse, par exemple le syntagme soey-ikin-ikin-i (esprit-pluriel-voir-voir-non.assertif) devient soey-kini-kini, le morphème *kini n'existant pas. Au niveau lexical, la langue chamanique use de mots « inconnus ». Certains sont inconnus de tous, même des chamanes, comme le terme măgoratiğ, leitmotiv auquel nul ne sut donner un sens. D'autres, dans des développements, peuvent recevoir un sens hypothétique, inféré, par les chamanes, d'un contexte très large. Ainsi, par exemple, l'hapax motopkor reçoit le sens de «cuirasse de résine » dans l'énoncé motopkor yesapẽ yemani opiekamidna, 
« revêtir mes pieds d'un motopkor » d'après un épisode mythologique où un personnage s'enduit les pieds de résine. Certains mots subissent un changement de valeur aspectuelle : - $a k \tilde{l}$ désigne ainsi un état (" être grand ») et non plus un processus («grandir»). Des particules syntaxiques subissent des altérations systématiques : le suffixe datif -kay devient -kayba, l'ablatif -pi devient -piba. L'usage des suffixes classificateurs est brouillé : ainsi dans le syntagme tamarikihr-e-xeb (agami-blanc-conjonction-feuille), -seb, « feuille », se substitue à -sin, « poil, plume », donnant le syntagme inusité « feuille d'agami ». Enfin, au niveau syntaxique, certaines particules sont systématiquement surajoutées comme le démonstratif lointain ye- ou l'itératif -pere-, tandis que d'autres sont supprimées, comme les anaphoriques ee- et yed-. Ainsi l'énoncé :

Ena-ma ye-pere yeka te te o-ya tiga o-mere-sabatẽ

anaphorique-causatif [=inviter] démonstratif-itératif conjonction intensif intensif 1sg-non.testimonial conjonction 1sg-itératif-partir

serait en langue ordinaire :

*Ena-ma yed-eka te o-ya ee-tiga o-labatẽ

anaphorique-causatif [=inviter] anaphorique-conjonction intensif 1sg-non.testimonial anaphorique-conjonction 1sg-partir

Tu [m’y] as invité [à] cela sans relâche alors même [que] je partais sans relâche

S'y ajoutent des modifications de l'ordre des mots : l'expression mere-oy-kasaba (itératif-homme-datif-imperfectif), complément courant du syntagme précédent, serait en langue ordinaire oy-esaba-ka (homme-imperfectif-datif), « vers là où l'homme se trouvait ».

L'obscurcissement du sens induit par ces procédés linguistiques est renforcé par l'usage de procédés logiques, qui produisent des énoncés auxquels aucun sens non ambigu ne peut être attribué. On rencontre, dans les chants chamaniques, des contradictions de divers ordres. Les plus évidentes sont de simples contradictions in adjecto, telle l'expression ğara-peh-kata (forêt-espace-couper) " enclore la forêt ", c'est-à-dire, dans le contexte suruí, " encercler l'environnement ». Plus perturbantes pour les auditeurs sont sans doute les antinomies portant sur les déictiques personnels. Dans cette catégorie, on relève non seulement des énoncés contradictoires, du genre pa-năg-ey o-or (1pl.incl-ancêtre-pluriel $1 \mathrm{sg}$ venir) « moi l'un de nos ancêtres à nous tous je viens », mais aussi des phénomènes d'inclusion paradoxale de l'auditeur dans des énoncés l'excluant : tout locuteur du suruí ne peut manquer de se considérer comme destinataire d'un discours où il identifie sans ambiguïté le pronom de la première personne inclusive $\mathrm{du}$ pluriel pa-, extrêmement récurrent dans ces énoncés qu'il ne comprend pourtant pas, paradoxe dont le sommet est l'expression typique des chants chamaniques pa-mere-ye-koe (1pl.incl-itératif-démonstratif-voix), " notre langue "toujours" à nous tous ». Cette difficulté d'identification des référents des déictiques se trouve 
souvent renforcée par l'indiscernabilité entre les énonciateurs potentiels du discours chamanique, l'esprit et le chamane. Les chants sont supposés être le discours direct de l'esprit, mais celui-ci y apparaît parfois à la première et à la troisième personnes dans la même phrase : l'esprit nommé Kadorotih, «Plumeau », chante :

o-ya ye-bona pa-năg-ey Kadoro-tih ye-mamner ka-xiter pa-mere-ye-koe ye-mãri

1 sg-non.testimonial démonstratif-effort 1 pl.incl-ancêtre-pluriel plumeau-esprit démonstratif-intensif datif-intensif 1pl.incl-itératif-démonstratif-langue démonstratifparler

Je me suis efforcé, moi, l'un de nos ancêtres à nous tous, ce Plumeau, de lui parler en personne dans notre langue de toujours à nous tous

L'esprit supposé parler par la bouche du chamane y décrit ce que, de fait, le chamane entend faire en le faisant parler. Enfin, certaines contradictions sont de nature performative, principalement par l'usage d'énoncés autoréférentiels, autoréférentialité souvent dénotée par des locutions adverbiales comme dans ce cas :

Enama yepere yeka te bokosa tiga omerealape yeoga wá-xiter (parler-intensif) tiga yoikãr yesodug meykayba

Tu m'as invité à faire cela et à ce moment-là même je n'ai pas fermé l'œil jusqu'à cette aurore-là en disant vraiment ce que je dis maintenant même et j'ai secoué les palmes pour vous

En outre s'ajoutent à ces procédés linguistiques et logiques des procédés sémantiques qui enrichissent et complexifient le sens - pour autant que l'auditeur ait surmonté les obstacles précédents. Outre l'évocation d'objets inconnus - tamarikihr, « agami blanc »-, ayant parfois un sens métaphorique - wakarob, " héron rouge », c'est-à-dire « ara »-, on rencontre des expressions dont il est difficile d'élaborer une interprétation pertinente, comme me-ey-ayxoh (cheminpluriel-éteindre), "éteindre des chemins ». Certaines locutions semblent même relever du jeu de mots, sur le principe du calembour, tel le qualificatif ğapomnih-

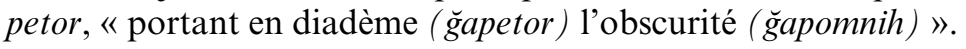

L'incompréhensibilité des chants chamaniques n'est cependant pas totale. En effet le nombre limité de ces figures finit par en rendre certaines au moins partiellement maîtrisables, à la manière d'un code assez basique. Certaines expressions très récurrentes peuvent être assez facilement décodées : beaucoup d'auditeurs ordinaires savent que la locution mere-oy (itératif-homme) désigne le chamane ou que le terme kador se substitue systématiquement au terme narayip, « plumeau rituel ». De même, par un raisonnement en termes d'oppositions contrastives, la plupart des adultes savent déduire que les expressions pamaihkinima et păgattih, apparaissant respectivement et systématiquement dans les chants des esprits aquatiques et des esprits célestes, ont trait l'une à l'eau (« notre eau infinie à tous ») et l'autre au ciel («notre ciel à tous »). Une part 
considérable des procédés détaillés précédemment, qui s'appliquent à un petit nombre d'éléments ensuite combinés pour former les énoncés des chants, peut ainsi être partiellement décodée. Cette limite à l'inintelligibilité de la langue chamanique est nécessaire pour en faire une compétence vérifiable, sur laquelle un certain nombre de gens - au minimum l'ensemble des chamanes - peut s'accorder pour reconnaître un chant comme conforme et donc chamanique. Cette vérification intéresse tous les auditeurs car, si la vindicte qui menace les mauvais chanteurs est celle des esprits, nul ne souhaite bien sûr employer un mauvais chamane à son service.

Au regard de ces procédés d'obscurcissement du langage ordinaire et de leur champ d'application, le chant chamanique pourrait sembler se réduire à une technique de codage sommaire. De fait la langue chamanique diminue considérablement le nombre d'énoncés possibles : pour rester " compréhensibles », c'està-dire «traduisibles » en langue ordinaire, le nombre de métaphores et autres torsions doit rester limité. Cette langue tendrait à se réduire à une combinatoire avec un nombre relativement faible d'éléments. Il est donc surprenant que les Suruí persistent à décrire le pouvoir du chamane, non comme l'acquisition d'un nombre fini d'objets, mais comme l'accès à une compétence ayant un champ d'application potentiellement infini. En effet, la notion de «cœur » (agõa), à laquelle on fait appel pour désigner toute activité chamanique en tant que telle, dénote en suruí tout ce qui relève de l'intériorité, de l'inaccessible à autrui et donc de l'indétermination. La notion polysémique de -koe (« voix, langue, genre, ton, air »), utilisée pour décrire le discours chanté des chamanes, implique certes une réduction du champ sémantique couvert par un " genre » discursif (les esprits ne peuvent « dire n'importe quoi », pas plus que les chanteurs humains lors d'un rituel festif). Cependant cette notion désigne toujours une forme expressive, une " langue », qui ne peut être réduite à un unique énoncé ${ }^{7}$. Sans spéculer excessivement sur le concept de « langue » des Suruí (qui ne disposent pas d'autre terme que -koe pour désigner leur langue ou celle des étrangers), on peut se demander si le -koe chamanique mérite cette qualification : est-il plus que la somme des énoncés du nombre limité des chants connus ? permet-il encore de produire des énoncés à l'infini ? ou ne fait-il que répéter des énoncés fixes ? Questions délicates, puisque les Suruí affirment aussi refuser toute innovation en matière de chant chamanique. Or l'ensemble des chants chamaniques n'est pas un ensemble clos, notamment du fait de la distinction entre leitmotiv et développement. Dans les développements, une fois respectées certaines exigences qui attestent de la compétence linguistique à produire des énoncés susceptibles de passer pour chamaniques, on peut, dans une certaine mesure, recourir à la langue ordinaire ou à des structures tirées de celle-ci. Par exemple, à partir du terme tororo, idéophone substitut de mõbo, « cascade », un chant peut former le syntagme tororo-iway-ey (idéophone-maître-pluriel), " maîtres du glouglou » (i.e. autres esprits), sur le modèle de mõbo-iway-ey (cascade-maître-pluriel), "maîtres de la cascade », 
locution désignant ordinairement les ennemis humains. Quant aux leitmotive, où le sens compte peu, puisqu'il s'agit d'une simple identité, d'une sorte de nom propre lyrique, il existe une possibilité de les multiplier presque à l'infini, par des modifications minimes, portant notamment sur les idéophones. Ainsi l'esprit Ikõrnih, " Aigle-harpie », chante kattih xõrũ xõrũ, kattih xõrũ oğoy ; son « cousin » Ikõrud, « Petit Aigle-harpie », s'en distingue uniquement en substituant à l'idéophone xõrũ, «glatir », l'idéophone wuga, «battement d'ailes » dans son leitmotiv: kattih wuga, wuga, kattih wuga oğoy. Une dose d'" invention » de nouveaux esprits par ce procédé n'est pas à exclure.

Sous ses proclamations de fixité et de répétitivité - indéniables -, le chant chamanique paraît contraint de maintenir une certaine inventivité et des possibilités de renouvellement. En effet, privée de cette ouverture, la prétention à être « compris d'un coup » comme maîtrise pleine et entière d'une compétence linguistique, qui fait la spécificité de l'activité chamanique et la légitimité des chamanes, ne pourrait être maintenue : on pourrait aisément apprendre les chants un par un. La « langue » chamanique n'est donc que la résultante de la tension entre la nécessité de conserver une possibilité de vérifier une compétence, d'où découle une exigence de semi-compréhensibilité, et le refus de toute possibilité d'apprentissage initiatique.

\section{LE STYLE DES CHANTS « HUMAINS »}

Visant à la singularité et à la perpétuation de son souvenir, le chant humain ne peut, lui non plus, se dispenser d'une certaine distinction par rapport au langage ordinaire, ce qui induit une opacification de son sens. Ici, seul le style peut jouer ce rôle de saillance : le contenu narratif d'un chant reste extrêmement pauvre et, concentré dans les développements, ne permet guère de distinguer un chant d'un autre au sein d'un même genre. Or cette exigence d'originalité doit être satisfaite au moyen d'un répertoire assez restreint, afin de rester conforme à un genre, défini par un petit nombre de métaphores contraintes. Dans ce cadre, l'originalité peut être produite de diverses manières. Dans les développements, les métaphores, bien qu'assez contraintes, s'engendrent parfois les unes les autres. Ainsi, à partir de la métaphore quasi obligatoire $i k \tilde{r} r$, " aigle-harpie », oiseau nidificateur, dans un chant de fête de construction de maison, d'autres métaphores ornithologiques, plus rares, peuvent parfois apparaître, comme kasar, " ara », entièrement immotivé dans ce contexte. Dans les leitmotive, on rencontre fréquemment des déformations phonologiques et des formules syntaxico-sémantiques idiosyncrasiques. Par exemple, l'énoncé onáhm, constituant presque à lui seul le leitmotiv d'un chant de fête d'essartage, réunit ces deux phénomènes : il s'agit d'une altération phonologique du syntagme $o$-náğ (1sg-tenir) "me tenir à la main », énoncé auquel il semble d'abord difficile d'attribuer un quelconque sens, avant que 
la suite du chant ne révèle qu'il faut y voir un jeu de mots condensant irrégulièrement o-nãbe-txáğ (1sg-hache-tenir) " tenir ma hache»-on aurait dû avoir $i$-txáğ (3sg-tenir) -, signifiant donc en quelque sorte « me tenir moi et ma hache en main [à son service] ». Ce cas répond pleinement à l'idéal esthétique qui régit ces innovations : la simplicité de la formule «bien trouvée », à la fois concise et sémantiquement riche.

Toutefois l'étude d'un grand nombre de chants, tant « beaux » que médiocres, révèle rapidement un style qu'on peut qualifier, d'un point de vue formel, de maniériste. Cela se marque par l'usage d'un registre lexical «soutenu », entièrement compréhensible, mais très peu usité dans le langage parlé ; ainsi les « ennemis » sont toujours désignés par le terme loykubey, vocable limpide, mais auquel le langage parlé préférerait lahdey. Le style chanté affectionne les périphrases, les tautologies et les génitifs autoréférentiels. Des expressions comme ğaratih-mă̆a-ba (grand.essart-faire-nominalisateur), "chose qui fait de grands essarts » (pour nãbea, « hache »), meko-ne-pami-b (jaguar-objet.secondpeur-nominalisateur), " chose qui effraie le jaguar » (pour yab, «flèche ») ou ih-iway-ma-ih (bière-maître-possessif-bière), " bière du maître de la bière " sont caractéristiques de ce style. Cela peut produire des sortes de jeux de mots :

Ikabi-ma-ih-kabi (mortier-possessif-bière-bénéfactif) eya lakatipereitxa te omaor a, omaor $i$

Pour la bière de « ce qui est pour la bière » [mortier], tu m'as fait venir la faim au ventre, dit-on, tu m'as fait venir, n'est-ce pas

Cette tendance à la complexification syntaxique produit des énoncés comportant un bien plus grand nombre de subordonnées que le discours ordinaire (hypotaxe) :

[Nan-ena bo-ğa, [ana bo-mere-ya sona], [ [o-ma-ur-popi-ğar-e-maki sona po yedemi-ke] o-ya o-ma-xiener xa-ke-yã], [o-kere-ibita yã ], o-ya ena yã ]

[interrogatif-anaphorique [1sg-datif démonstratif $1 \mathrm{sg}$-itératif-non.testimonial souvent] [[1sg-possessif-arc-victime-chercher-conjonction-faire souvent intensif comme-pensée] 1sg-non.testimonial 1sg-causatif-prestigieux intention-pensée-non.testimonial] [1sgdormir-sous non.testimonial] 1sg-non.testimonial anaphorique non.testimonial]

Pourquoi, moi, de ce que je faisais toujours, j’y ai songé, songé que j'étais encore prestigieux, comme lorsque je traquais sans répit la victime de mon arc, pendant mon sommeil, dit-on?

Ce maniérisme mène, dans certains cas, à une réversibilité quasi totale des métaphores, ainsi qu'en atteste la symétrie entre ce chant d'essartage [E], où le pécari, figure topique de l'ennemi, désigne l'arbre à abattre et ce chant de guerre $[\mathrm{F}]$ où la hache et l'arbre tiennent lieu de flèche et d'ennemi :

[E] Ohgõakarniga meparayakadna mamẽbena yepi $i$ Omaurpereka aar, aar oğabi 
L'angoisse a étreint mon cœur, de crainte de ce qu'il allait faire ensuite, cet ouvreur de piste, ce pécari, n'est-ce pas

Il est tombé par le fait de mon arc, il est tombé pour moi

[F] Kanater ğaratihmăgaba ma aĩ

Kanater onãbe ma aĩ ippóyhka aĩ aĩ oğabi

Jusqu'où s'est-elle enfoncée la chose qui fait les grands essarts

Jusqu'où s'est-elle enfoncée ma hache, dans le grand tronc, pour moi

Du point de vue de leur forme linguistique et stylistique, les chants chamaniques semblent constituer un modèle implicite et inavoué que les chants humains n'arrivent jamais à égaler. Comme s'ils caressaient le rêve d'aboutir aux mêmes résultats que la langue chamanique - produire des objets aussi saillants et mémorables -, les chanteurs humains tentent de suppléer aux limites de leur inspiration en appliquant le même principe (torsions et altérations du langage ordinaire). Ces écarts doivent rester moindres, car ils ne peuvent se permettre de courir le risque de l'incompréhension - dans la mesure où ils doivent tout de même revendiquer des faits. Aussi, recourant à un petit nombre de techniques connues de tous, réussissent-ils rarement à produire des énoncés radicalement originaux.

\section{PragmatiQue : IRONiE ET INSOlenCe}

En revanche, sous l'angle pragmatique, les chants humains semblent mettre en œuvre une technique spécifique, s'écartant nettement des usages de l'énonciation ordinaire, en particulier par le type de relation qu'elle induit entre chanteurs et auditeurs. Ces chants se caractérisent par une structure énonciative complexe, qui affecte le jugement que les auditeurs peuvent porter sur le statut de ces énoncés, sur leur légitimité et sur le rapport entre ces énoncés et leurs énonciateurs. Le chant humain exploite largement les possibilités offertes par l'usage obligatoire du non-testimonial ya, qui englobe tout énoncé dans un " on dit » général. Nombre de chants renforcent ce désengagement de l'énonciateur et le complexifient par un enchâssement de citations de discours : on rencontre fréquemment des structures du type « on dit que je dis que j'ai fait », voire plus complexes, comme " on dit que je dis qu'il dit que j'ai fait». Cela permet des énoncés auto-laudatifs qui se ménagent en quelque sorte une échappatoire au cas où ils apparaîtraient comme ridicules (Aikhenvald 2004, p. 226). En témoignent ces énoncés de chanteurs de prestige modeste, jeunes ou féminins :

" "Ye bo paladnadetare sakah $i$, garatihmağa te paneikin ena i", Ğurey ya oğay $i$ », te boliyã.

" "Oui, celui que nous connaissons bien va ouvrir un grand essart sous nos yeux à tous", les Blancs diraient-ils de moi », c'est ce que je dis, dit-on. 
"One ikõrey ya mana téhreh mammóyheperẽkabi te, asabamaã te ana téhreh, awemamoga te, "Ãka paba maloypóyhpamibeka palabamaã ma”" " awekay enareh, tasa enareh. «Ce n'est pas difficile pour les aigles-harpies de bâtir leur maison à la fourche du grand châtaigner du Brésil, de se rassembler pour dire "Bâtissons notre maison par crainte du déluge" ", qu'ils le disent, qu'ils le disent tous.

Par un jeu sur les inversions de point de vue ${ }^{8}$, l'enchâssement des citations permet de produire des effets d'équivoque - d'autant plus déconcertants que la marque de la citation se place en suruí à la fin de la phrase. Ainsi, dans ce chant, l'auditeur ne découvre qu'au bout de trois minutes (sur six), que tout le discours qu'il a entendu jusqu'alors était énoncé du point de vue de l'ennemi :

" "Alaatemiter, alaatemiter" oliyã, te oliyã... "Alaatemiter" oliyã », yaka makoy $i$.

«"J'ai failli mourir, j'ai failli mourir", dis-je, oui dis-je, dit-on... "J'ai failli mourir", dis-je, dit-on », doit-il [= l'ennemi] dire là-bas

Cette même technique d'enchâssement peut engendrer un effet ironique - exploitant d'ailleurs une technique de plaisanterie courante chez les Suruí, la moquerie par citation du moqué. Cette ironie est particulièrement utilisée par les femmes qui se risquent à chanter :

" Ana toyhxiyã » te ihiwayaihkanẽyey oğay ekanan ne kaledma ihiwayaihkanẽyeyibebnota te tamaihkabi oĩ ma ena, oma wa, oma ena.

«C'est ainsi que nous autres faisons, dit-on » oui, bien que ceux qui désirent la bière du maître de la bière ne m'aient pas invitée ainsi, moi je suis entrée en le disant là où se trouve leur bière, à leur suite, ceux qui désirent la bière du maître de la bière, moi, oui, moi je l'ai déjà fait

Parmi les effets produits par l'usage de citations, on peut enfin noter l'ambivalence qu'induit l'insistance sur le statut du discours rapporté en tant que discours, pensée et représentation. Cela peut aussi bien signifier l'écart entre le discours et les faits que la puissance de l'intention modelant la réalité. Cette ambivalence se voit en particulier par le large usage d'un adverbe, kaled, qui problématise le rapport de l'énonciateur à son énoncé, avec un sens ambigu, allant de « il l'a dit à juste titre », jusqu'à " il n'a fait que le dire ». Ce terme reste toujours difficile à traduire, nécessitant généralement une glose tirée du contexte :

" Paba agota eetiga ãikahbaã eid masayeyikahbpena eidğa yedeka ĕgay », kaled ola ikayã.

Si nous allons prendre ces dents demain, alors nous te [fournirons] un collier de dents de singe à toi pour ta [femme], lui ai-je dit, moqueur [à un chasseur incapable], dit-on.

Ete « Kixana ya iñan iter $i$ » kaled ola tar ã.

«Ce martin-pêcheur est un vilain » me répété-je amèrement, dit-on.

"Iwekũyikin e oliyã " kaled tasa awekay $i$.

«Cela me plaît » se disent-ils déjà, dirait-on. 
" Meyakadegeypi ŏgay ma » kaled ola takayã.

«Donnez-moi de vos larves » leur dis-je sans espoir, dit-on.

Ana bomereya sona, omaurmixakorka te omaurtáğ iter sona po yedemike oya omapağawarõm ne ke omaurtáğ iyã, oya ena yã, kaled oya ena yã.

Ce que je faisais toujours, j'y ai songé, que j'étais [encore], arc en main, respecté de tous comme lorsque j'étais à la poursuite du tamanoir de mon arc, mon arc bien en main, oui dit-on, oui dans mon rêve dit-on.

Cette formulation ambiguë, cette énonciation équivoque et ce ton ironique des chants humains impliquent que leur tonalité générale, laissée partiellement à l'interprétation des auditeurs, peut osciller dans des nuances allant de la morgue à l'autodérision. Cette ambivalence ne va pas sans difficultés. L'ironie est un art qui exige une certaine modération pour ne pas tomber dans ce qu'on pourrait qualifier de bouffonnerie ${ }^{9}$, comme le montre le cas de chants jugés " ratés », tel celui-ci :

" "Ano yakade mixağariga akerõm anie sakah i”, Ğurey ya oikin ena $i$ », te boliyã.

«"Voilà celui qui ne dort jamais de la nuit [= fait l'amour toute la nuit]", les Blancs diraient-ils en me voyant », c'est ce que je dis, dit-on.

À rebours de ce que l'analyse montrait en matière linguistique et stylistique, sous l'angle pragmatique, ce sont les chants humains qui semblent offrir un modèle aux chants chamaniques. En effet les chants humains reposent sur une disposition assez simple : une opposition énonciateur $v s$ auditeur, médiatisée par un " on dit », l'énonciateur cherchant à faire admettre au destinataire la vérité d'énoncés portant en dernière instance sur lui-même en les attribuant à ce « on dit » indéfini. Or les chants chamaniques complexifient ce face à face, du fait de l'ambiguïté propre à la langue chamanique. L'énonciateur y est ambigu - chamane ou esprit ? Le destinataire y reste indécidable - chamane ou patient ? L'auditeur y est à la fois nécessaire - comme patient ou commanditaire - et nié - par l'inintelligibilité. Étant donné les conditions d'énonciation - risque « mortel » pesant sur le chanteur - et les spécificités linguistiques de ces chants semiincompréhensibles, cette configuration induit un registre chamanique distinct de celui des chants humains. À l'ironie laissant ouvertes les hypothèses de la morgue ou de l'autodérision, se substitue ici ce que, faute de terme mieux approprié, on pourrait qualifier d'insolence, narquoise et détachée : le chant suggère à l'auditeur des débuts d'interprétation, mais lui refuse un statut de destinataire et sape la légitimité de son interprétation, ou du moins de celle qu'il tenterait d'élaborer seul. Au lieu du « Vous savez ce que je dis et vous savez que vous ne pouvez le contredire parce que vous savez que cela pourrait vouloir dire autre chose » du chant humain, le chant chamanique propose un «Vous savez un peu ce que je dis mais vous savez ne pas pouvoir le dire ". C'est donc, semble-t-il, l'écart par rapport au langage ordinaire qui, par son degré et ses modalités, produit deux 
types d'attitudes spécifiques des auditeurs par rapport aux énoncés chantés: soumission à l'autorité du chant chamanique, contestation continuelle de la perfection du chant humain ${ }^{10}$.

\section{EFFICACITÉ ET FONCTIONS DU CHANT}

On pourrait s'attendre à ce que ces attitudes aient un écho sur les fonctions de ces chants, notamment sur la présomption d'efficacité, thérapeutique ou autre, du chant chamanique - puisque c'est souvent un des critères motivant cette catégorisation au sein des genres lyriques. Or, pourtant, l'efficacité n'apparaît pas vraiment comme un caractère du chant, au sens où son énonciation serait susceptible de produire divers effets, bénéfiques ou nuisibles. Au contraire, non seulement les chants humains sont énoncés postérieurement à l'acte - ingestion de bière, meurtre, etc. - qui les motive, mais même les chants chamaniques semblent inutiles ou superflus aux fins explicites des activités dans lesquelles ils sont chantés : l'efficace de la cure est considérée comme reposant d'abord sur d'autres actes du chamane - ivresse tabagique et insufflations -, tandis que certaines activités constitutives de la puissance chamanique, comme l'agression magique, ne requièrent aucune énonciation verbale. Il existe d'ailleurs un statut de guérisseur silencieux (koro), distinct de celui de chamane (wãwã), décrit comme « quasi-chamane » (wãwã-kõ $b$ : chamane-presque), résultant d'une élection inaboutie. Celui-ci, qui ne maîtrise pas la langue chamanique et ne chante pas, exclu des rituels collectifs, ne traite les maladies que par une succion locale et muette, en état d'ivresse tabagique. Il est néanmoins réputé posséder un pouvoir thérapeutique équivalent, voire parfois supérieur à celui du chamane accompli et chanteur. Enfin, s'il y a un domaine où, chez les Suruí, on peut parler d'une évaluation " esthétique ", au sens d'indépendante de critère d'utilité, c'est bien celui des chants chamaniques. Certains d'entre eux sont déclarés particulièrement «beaux» ou «bons en tant que chants» (ta-koe-paor iter: 3pl-voix-bon intensif), sans corrélation aucune avec la bienveillance et les vertus thérapeutiques des esprits qui en sont les auteurs et propriétaires. Ainsi le chant de l'esprit Winnih, très malveillant, est unanimement jugé magnifique, à l'égal de celui d'Ããritih, sans malice mais peu efficace, et au contraire de Mokóhba, entité dénuée d'animosité et au chant sans grâce, et d'Ikabitih, peu mélodieux et funeste.

Commentaire redondant ou postérieur à l'action, le chant ne semble avoir de prise que sur son inscription dans le temps. Le chant implique en effet un rapport spécifique au temps, en particulier par la constitution d'événements s'opposant à la routine de l'existence villageoise. Cela se remarque d'abord dans l'usage fréquent des chants humains comme repères temporels. Ceux-ci servent en effet de jalons «historiques» permettant d'ordonner les événements les uns par rapport aux autres : une anecdote - chasse miraculeuse, blessure, querelle, etc. - 
sera souvent située dans le passé par le cycle festif au cours duquel elle a eu lieu et par un chant qui y a été produit. Lorsqu'un chant est rechanté, un auditeur qui avait assisté à son énonciation initiale saura toujours indiquer " quand » celle-ci a eu lieu - i.e. l'âge qu'il avait, le lieu, le «maître » de la bière consommée, les autres chants chantés avant et après. Le rôle de jalon du chant n'apparaît probablement nulle part mieux que dans la toponymie - celle-ci est un instrument de datation, permettant de situer les événements en fonction du village où l'on résidait lorsqu'ils ont eu lieu. Alors que les villages récemment abandonnés sont dénommés d'après des traits matériels (végétation endémique, configuration des maisons, etc.), les désignations de villages anciens, dont la localisation spatiale est très incertaine - en particulier ceux dont tous les résidents sont aujourd'hui décédés -, sont souvent tirées de métaphores jugées originales d'un chant qui y a été composé : Yoykapsiwaba, "Lieu où l'on but du jus de patauá », Lorğapsiwaba, « Où l'on but du jus de lorğap » (n. i.), Marãyasiwaba, « Où l'on but du jus de canne à sucre », etc., chacune de ces boissons étant une métaphore de la bière inventée et chantée lors d'une fête en ces lieux.

Le rôle des chants humains ne se résume pas à un simple usage de repère. L'énonciation lyrique marque aussi l'accomplissement, la conclusion d'une série d'actes - au minimum, la mise au défi de la production de bière, la production de biens de compensation de celle-ci (colliers, flèches, viande) et, enfin, sa consommation. Aussi chaque genre lyrique est-il désigné par l'activité que la fête de

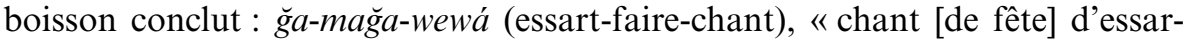
tage ", mokãy-kar-ewá (feu-chercher-chant), " chant [de fête] de ramassage de bois », ğer-mabi-ewá (chasser-locatif-chant), "chant [de fête] de retour de chasse », etc. La postériorité du chant à son activité motivante s'inscrit dans une éthique générale de la lenteur et de la patience propre aux Suruí. Le propre d'un grand homme est de savoir attendre le moment opportun pour prendre une initiative et réaliser sans délai son objet, sans avoir pris le risque de dévoiler des désirs irréalisables qui attesteraient d'une part de faiblesse et d'impuissance - et non pas d'élucubrer des projets, de sombrer dans la vantardise velléitaire et vaine inhérente à la jacasserie féminine. Chanter après et, pour ainsi dire, "sur le socle » de l'événement accompli s'oppose au bavardage stérile précédant, différant, voire entravant, l'action fantasmée ${ }^{11}$. Ainsi la métaphore de prédilection des chanteurs pour s'auto-décrire est l'aigle-harpie, incarnation par excellence de la " grandeur» : tueur planant silencieux et impassible, dont la patience infinie n'a d'égale que l'action foudroyante. Dans un récit quelconque, le rappel du chant d'une fête pouvant apparaître comme l'aboutissement d'une série d'anecdotes sert d'ailleurs souvent de conclusion ou, tout au moins, de butée, sur laquelle le narrateur s'arrête, avant éventuellement de passer à une autre série narrative. On notera enfin qu'a contrario il existe tout un ensemble de (non-)événements qui ne peuvent être chantés, c'est-à-dire de faits qui, pour saillants qu'ils puissent paraître à un regard étranger, n'ont pas semblé aux Suruí dignes d'être 
célébrés : citons ainsi la naissance, le mariage, la mort. Ce dédain s'explique parce qu'il s'agit là, pour les Suruí, non pas d'accomplissements, mais d'amorces de processus - en l'occurrence, de formation d'individus, de familles, d'esprits ancestraux.

Il semble toutefois que cette analyse sied mal aux chants chamaniques. Ceux-ci, répétés quasiment à l'identique à chaque énonciation, semblent peu soucieux de distinguer des événements. Leur temps apparaît comme une durée distendue et exposée ; ils ont plutôt tendance à décrire des processus répétitifs que des actes isolés. Plus exactement, ils décrivent des événements relativement cruciaux - la venue de l'esprit au chevet du malade - comme des processus répétitifs. En atteste l'omniprésence d'une particule, -pere-, de valeur itérative, qui s'infiltre dans nombre de syntagmes de la langue chamanique, bien au-delà de sa fréquence ordinaire :

Ena-ma ye-pere yeka te te o-ya tiga o-mere-sabatẽ mere-oy-ka-saba.

anaphorique-causatif [=inviter] démonstratif-itératif conjonction intensif intensif 1sg-non.testimonial conjonction 1sg-itératif-partir itératif-homme-datif-imperfectif.

Tu as invité à cela sans relâche alors même je partais sans relâche là où se trouvait l'homme sans relâche.

Paradoxalement, ce trait contamine fortement les chants humains, qui font eux aussi un large usage de la particule -pere-, ce qui pourrait sembler contradictoire avec le rôle qu'on vient de leur attribuer - paradoxe qu'on ne peut traiter à la légère, dans la mesure où ladite particule vient se nicher jusque dans le terme désignant la spécificité de l'énonciation lyrique : merewá (itératif-parler)!

Cette combinaison paradoxale d'une fonction de césure événementielle et d'une forme essentiellement répétitive s'explique dès lors qu'on prend en compte une autre dimension de l'accomplissement que dénote le chant, humain en particulier. Le chant est l'achèvement de l'individuation de l'individu (à laquelle contribuent bien d'autres facteurs, ses actes en premier lieu). L'énonciation lyrique dans le contexte festif est non seulement la preuve de la capacité d'un homme sinon à diriger, du moins à être accepté, au sein d'une faction rituelle, mais encore, en tant que composition d'un chant original, la démonstration de sa capacité à se singulariser au sein de ce collectif qui l'a accepté ou suivi. Le chant en tant qu'objet est la substance même de l'individu, son élément le plus durable : c'est par ses chants qu'on évoque un homme décédé - l'énonciation des noms personnels des morts est prohibée - et qu'il subsiste dans le souvenir de ses descendants. Les chants humains ne se limitent pas à dénoter la singularité de leur auteur par leurs différences stylistiques : à travers leur description d'un événement comme processus répétitif, ils paraissent viser, à partir de cet acte, à constituer ce haut fait en (auto-)définition de l'individu. Cet acte ponctuel et la manière de l'énoncer deviennent l'indice d'un mode d'être propre, celui de sa grandeur individuelle : 
Ye te o-ya o-ma ma-mokãy-ariga te o-mere-de sona epitxa te e-ğa-yã o-mere-kanẽ e-marija-be-maki e-ğa-yã, ye te wa ena e-ğa-yã.

démonstratif intensif $1 \mathrm{sg}$-non.testimonial $1 \mathrm{sg}$-causatif indéfini-feu-chaque intensif $1 \mathrm{sg}$-itératif-testimonial souvent conjonction intensif $2 \mathrm{sg}$-datif-non.testimonial $1 \mathrm{sg}$ itératif-vouloir 2sg-ivre-conjonction-faire 2sg-datif-non.testimonial démonstratif intensif exhortatif anaphorique 2 sg-datif-non.testimonial.

Je te dis que moi je t'ai enivré, toi qui aimes [ma bière] sans relâche, parce que je suis celui qui a sans relâche et toujours ramassé du bois sec de chaque espèce, je te le dis, dit-on.

Évoquer un événement relativement ancien, c'est donc concrètement citer les chants de son ou ses protagonistes, ce qui revient à décrire ces derniers selon leur style propre, en attribuant à chacun une manière d'être stable, idéale et singulière. Or, comme ces événements sont en tant que tels tous très semblables - il ne s'agit jamais que de boire de la bière -, ce qui subsiste dans la mémoire des participants, des spectateurs ou de leurs descendants, ce sont les chants dénotant et décrivant des individualités. Cette efficacité individuante est le propre du chant en tant que forme : si le chant humain est agent d'individuation du chanteur, c'est aussi le cas du chant chamanique, puisque le chant constitue presque toute la réalité de l'esprit auteur. Les descriptions morphologiques ou éthologiques que les chamanes peuvent donner des esprits sont assez pauvres et instables. Leur nom même, éponyme d'une espèce naturelle, s'explique souvent par le contenu du chant, tout lien «réel» (engendrement, élevage, etc.) avec les êtres naturels étant systématiquement refusé. Ainsi la seule motivation du nom de l'esprit Mekopebabtih, "Martre » (Eira barbara) est qu'il se décrit, dans son chant, comme lascif et amateur de miel.

\section{DESTIN DES CHANTS ET DES CHANTEURS}

Cette fonction individuante du chant, s'appliquant tantôt à l'auteur humain, tantôt à l'auteur spirituel, permet de comprendre la fortune mémorielle des chants et la destinée sociologique des chanteurs de chaque genre. D'un point de vue sociologique, le destin des chanteurs humains semble tendre vers le silence, ou du moins la réserve, attitude qu'adoptent les plus prestigieux d'entre eux. Dans la mesure où il prétend démontrer le savoir et énoncer la grandeur des actes de son auteur, le chant humain expose inévitablement cette réputation au risque d'être contestée, discutée ou rabaissée : tel chant était-il aussi beau que tel autre ? Les actes accomplis méritaient-ils une célébration si tapageuse? L'excellence la plus évidente et assurée étant celle qui n'a pas à s'énoncer, l'achèvement du chant, au sens de son degré suprême, ou plutôt de la limite vers laquelle il tend, c'est donc logiquement le silence, lorsque la grandeur du grand homme n'a plus besoin d'être proclamée - d'où le silence relatif des "maitres de la lumière ». Inversement, l'excès de chant chez les jeunes gens friands de genres mineurs (i.e. motivés 
par des actes triviaux : chasse, rêve, etc.) équivaut à sa dévaluation. Du point de vue mémoriel, le destin des chants humains semble être le recyclage : être repris et rechantés à diverses échelles. À court terme, ils sont repris, comme jalons de récits ou dans un simple usage récréatif (divertissement au travail, par exemple), par tous ceux qui, parmi les auditeurs, en ont apprécié la beauté. Cette reprise est la preuve de la «célébrité » et de la reconnaissance pour laquelle rivalisent les chanteurs d'une même faction. À plus long terme, au-delà de la durée de vie du chanteur, ses chants lui survivent par ces reprises. Au cours de ces citations successives, qui peuvent en préserver certains sur soixante-dix, voire quatrevingts ans, les chants subissent cependant un processus de réduction, au terme duquel ne subsistera que leur leitmotiv - c'est presque tout ce qu'on peut reproduire des chants après deux générations d'écart. Enfin, lorsqu'au bout de deux ou trois générations, le souvenir du chanteur s'estompe de la mémoire de la plupart des auditeurs potentiels, les chants d'un mort finissent probablement par fournir un stock de figures, d'airs et de métaphores : une ressource dans laquelle ses descendants peuvent discrètement puiser leur inspiration. Ce recyclage sera d'autant plus légitime - moins contestable - que le plagiaire sera proche (en ligne agnatique) du plagié. De ce fait, on peut dire que le chant est non seulement le garant du souvenir des ancêtres, mais aussi leur substance même - d'où l'oubli nécessaire des ancêtres, comme auteurs propriétaires d'un certain nombre de styles, afin de pouvoir produire de «nouveaux» chants, c'est-à-dire de nouveaux individus.

Ce processus ne peut avoir lieu avec les chants chamaniques : ce principe d'oubli et de renouvellement est interdit par l'immutabilité des chants chamaniques, indéfiniment répétés à l'identique, directement inspirés par les esprits, empêchant ainsi l'individualisation du chamane en tant que chamane. En retour, cette immutabilité assure la constitution du groupe des chamanes en tant que collectif disposant de capacités spécifiques égalitairement possédées par ses membres, ce que proclame la théorie de l'accès à la compétence chamanique par voie d'élection. Aussi le chant chamanique apparaît-il comme un piège sociologique : par cette activité lyrique, on peut se faire soudainement reconnaître un statut non ordinaire, on peut en quelque sorte court-circuiter l'acquisition progressive et patiente du prestige du « savoir » à travers les fêtes et les chants, mais on s'enferme dans une technique qui tend à l'anonymat du chanteur et fait obstacle à son individuation. Un chamane ne laisse guère de souvenir en tant que chamane, s'il n'était pas aussi un grand producteur de chants humains. Les guerriers les plus prestigieux, ceux dont le souvenir subsiste plusieurs décennies après leur mort, se sont d'ailleurs souvent dispensés de maîtriser les pouvoirs chamaniques, construisant leur gloire sur leurs seuls exploits belliqueux et festifs. L'excellence du chamane (en tant que chamane) reste toujours inférieure à celle du grand homme chez les Suruí. Son activité est d'ailleurs toujours subordonnée, puisqu'il ne peut l'exercer qu'au service de l'homme fort de sa 
faction. Parallèlement, l'existence du chant chamanique reste toujours précaire, menacée par le pouvoir muet et efficace des koro, ces «quasi-chamanes » qui semblent prétendre, en se passant du chant dans leurs activités thérapeutiques ou agressives, pousser ce court-circuit à son degré ultime. Tout l'enjeu de la pratique du chamanisme, et en particulier la solidarité des chamanes dans les rituels collectifs et leur officielle égalité de puissance, est peut-être de conserver un espace propre, entre le chant humain qui n'a d'autre finalité que sa propre énonciation et l'efficacité directe et silencieuse du « quasi-chamanisme ».

Ces divergences de résultat entre ces formes lyriques et, au-delà, entre les destins de leurs énonciateurs, à partir d'une parenté indéniable, s'enracinent dans les possibilités d'écart par rapport au langage ordinaire que s'autorise chacun de ces genres et dans les usages que chacun en fait. En maximisant cet écart, le chant chamanique est condamné à la répétition à l'identique, donnant un pouvoir général et indifférencié entre tous les individus qui le pratiquent, formulé en termes de tout ou rien. En le minimisant, le chant humain suscite une exigence d'originalité et de renouvellement qui lui confère un pouvoir individuant et en fait un instrument au service de la grandeur personnelle. Dimensions sociologiques et techniques rhétoriques apparaissent ainsi intimement liées dans l'art lyrique suruí. *

* Manuscrit reçu en septembre 2009, accepté pour publication en janvier 2011.

\section{NOTES}

1. Les Suruí, ou Paiter selon leur autodénomination, contactés en 1969, vivent depuis sur la Terre indigène Sete de Setembro (Rondônia et Mato Grosso) et comptent aujourd'hui environ un millier d'individus. Divisés en quatre clans exogames démographiquement très inégaux, traditionnellement chasseurs et horticulteurs, résidant concentrés en un ou deux gros villages, leur mode de vie a subi de profonds changements depuis le contact avec la société brésilienne : désormais dispersés en petits hameaux, insérés dans l'économie d'une région de colonisation agricole, vivant de la culture du café et de l'exploitation forestière, convertis au protestantisme, ils ont largement abandonné les activités rituelles décrites ici. Les seuls travaux ethnographiques publiés à leur sujet sont ceux de Mindlin $(1985 ; 1995)$. On trouvera des informations sur les fêtes de boisson tupi-mondé dans les travaux de Dal Poz (1991) et sur leur musique dans ceux d'Ermel (1988), recueillies chez les Cinta-Larga, voisins orientaux des Suruí. Le corpus de chants sur lequel se fonde notre analyse rassemble une centaine de chants du type que nous désignons comme « humain » (recueillis auprès d'une vingtaine de chanteurs) et une cinquantaine de chants chamaniques (interprétés par un unique chamane). À l'exception d'un tiers du corpus humain, recueilli à l'occasion des festivités entourant la construction d'une maison, ces chants ont été recueillis hors contexte, à notre requête principalement, durant un terrain de quinze mois en 2005-2007 - dans le cas des chants humains, il s'agit alors toujours de chants ayant passé l'épreuve de la remémoration, parfois plusieurs décennies après leur composition. Ils ont été traduits mot à mot, avec l'aide d'informateurs et parfois des chanteurs eux-mêmes. Ce corpus, non seulement pour les chants humains, mais aussi pour les chants chamaniques, ne représente qu'une faible part de la somme des chants potentiellement disponibles. Toutes les analyses morphologiques et syntaxiques sont de notre responsabilité de non-linguiste. Je remercie Pierre Déléage pour la relecture de cet article, dont une première version avait été présentée au séminaire du Laboratoire d'anthropologie sociale en décembre 2008. 
2. La fête de bière n'est certes pas le contexte unique de la production de chants humains. Outre sa diversité interne en espèces de fêtes liée chacune à une activité particulière (essartage, ornementation corporelle, etc.), d'autres contextes peuvent être prétextes au chant, la guerre au premier chef, mais aussi la chasse ou le rêve. Nous traiterons cependant ici l'ensemble de ces chants et contextes comme un genre unique, dont l'unité réside dans deux traits : d'une part, tous ces contextes incluent une relation agonistique à des êtres extérieurs au groupe des chanteurs potentiels (producteurs de bière, ennemis, proies), d'autre part, les relations entre ces chanteurs prennent la forme de la rivalité et de la lutte pour la reconnaissance.

3. Cette idée est récurrente dans les traditions chamaniques tupi (Viveiros de Castro 1992, p. 224 ; Oakdale2005, p. 84).

4. Cette attitude se voit particulièrement dans un cas extrême : les tentatives féminines de chant. On incite parfois les femmes à se lancer dans cette activité masculine, tout en sachant qu'elles n'y arriveront pas, ne serait-ce que du fait de leur position et des métaphores contraintes des chants. Ces contraintes ne peuvent être contournées qu'à condition de parvenir à manier très audacieusement l'ironie, ce à quoi seules quelques femmes dotées d'une exceptionnelle assurance et souvent âgées se risquent.

5. Certains chants peuvent être occasionnellement accompagnés ou précédés par le son des flûtes.

6. Les chants chamaniques sont dépourvus des marques du discours rapporté, telles qu'on les trouve notamment dans la mythologie, et qui recourent à une combinaison des modes testimonial et non-testimonial («j'ai été témoin [de] du fait qu'on rapporte $[y a]$ ceci... »).

7. Même les -koe les plus rudimentaires sont susceptibles d'exprimer plusieurs significations : les cris des espèces animales (sobag-ey-e-koe : gibier-pluriel-conjonction-voix) peuvent en général exprimer tant le consentement à s'offrir au chasseur que le refus. Inversement, tout -koe, sauf peut-être la langue suruí ordinaire, conçue comme « notre langue à tous » ( $p a-\breve{g o e}: 1 \mathrm{pl}$.incl-voix), est restreint à un champ d'énoncés pertinents plus ou moins limité : il y a des énoncés suruí qu'on juge impossibles à formuler en portugais (yara-koe : Blanc-voix), faute de lexique adéquat.

8. Phénomène fréquent dans les chants tupi liés aux activités agonistiques (Viveiros de Castro 1992, p. 243 ; Fausto 2001, p. 353 ; Oakdale 2005,p. 126).

9. La justesse du ton d'un chant dépend de conditions qui sont en partie au moins préalables au chant. C'est en fonction de son statut, de la « grandeur » qui lui est reconnue, qu'un individu peut s'autoriser tel ou tel degré d'ironie ou de vantardise.

10. Pour un phénomène comparable, voir Sherzer (1974).

11. On objectera à notre analyse le cas des chants de départ à la guerre, à la chasse, de construction de maison, qui sont chantés « avant » l'événement. Outre le fait qu'à chacun de ces chants correspond un genre postérieur à l'acte en question, en général plus valorisé, il nous semble que, dans la mesure où ces " chants d'avant-acte » s'appliquent à des activités collectives, il faut les considérer comme des moyens de réserver une initiative ou autorité sur des événements à venir et subséquemment sur les droits à chanter postérieurement sur ces faits. Cette assise du chant sur l'acte définit aussi les genres lyriques masculins chez les Cinta-Larga (Ermel 1988, p. 136).

\section{RÉFÉRENCES CITÉES}

Aikhenvald Alexandra

2004 Evidentiality, Oxford University Press, Oxford.

DAL Poz João

1991 No país dos Cinta-Larga, mémoire de maîtrise, Universidade de São Paulo, São Paulo.

DÉLÉAGE Pierre

2009 Le chant de l'anaconda : l'apprentissage du chamanisme chez les Sharanahua (Amazonie occidentale), Société d'ethnologie, Nanterre. 
Desclés Jean-Pierre et Zlatka Guentchéva

2000 "Énonciateur, locuteur, médiateur», in Aurore Monod Becquelin et Philippe Erikson (éd.), Les rituels du dialogue. Promenades ethnolinguistiques en terres amérindiennes, Société d'ethnologie, Nanterre.

ERMEL Priscilla

$1988 \quad$ O sentido mítico do som: ressonâncias estéticas da música tríbal dos índios Cinta-Larga, mémoire de maîtrise, Pontifícia Universidade Católica de São Paulo, São Paulo.

Fausto Carlos

2001 Inimigos fiéis : história, guerra e xamanismo na Amazônia, Editora da USP, São Paulo.

MindLIN Betty

1985 Nós Paiter : os Suruí de Rondônia, Vozes, Petrópolis.

1995 Unwritten stories of the Surui Indians of Rondônia, University of Texas, Institute of Latin American Studies, Austin.

OAKDALE Suzanne

2005 I foresee my life : the ritual performance of autobiography in an Amazonian community, University of Nebraska Press, Lincoln.

SHERZER Joel

1974 «Namakke, Summakke, Kormakke: three types of Cuna speech event »,| in Richard Bauman et Joel Sherzer (éd.), Explorations in the ethnography of speaking, Cambridge University Press, Cambridge.

SURRALLÉs Alexandre

2003 Au cour du sens. Perception, affectivité, action chez les Candoshi, CNRS Éditions/Éditions de la MSH, Paris.

Viveiros de CASTRo Eduardo

1992 From the enemy's point of view, University of Chicago Press, Chicago. 